\title{
Contractile properties and fatigue of the diaphragm in man
}

JOHN MOXHAM, A J R MORRIS, S G SPIRO, R H T EDWARDS, AND MALCOLM GREEN

From the Brompton Hospital and University College Hospital Medical School, London

ABSTRACT We studied the pressure developed by the diaphragm in response to stimulation of the phrenic nerve in the neck, in three normal men. When the phrenic was electrically stimulated at increasing frequencies the diaphragm responded by increasing transdiaphragmatic pressure to give a frequency-pressure curve similar to the frequency-force curve for other skeletal muscles. The subjects then breathed through an inspiratory resistance for as long as possible and the frequency-pressure curve was repeated. It was found that the diaphragm developed low frequency fatigue, in the same way as previously described for other muscles. We conclude that the diaphragm has contractile properties similar to those of other skeletal muscles. Low frequency fatigue of the diaphragm could contribute to respiratory failure in patients with lung disease.

The diaphragm is the most important muscle of respiration. There have been few studies of the contractile properties of this muscle although the response to electrical stimulation has been studied in animals, ${ }^{1}$ and the human diaphragm has been investigated in vitro. ${ }^{2} 3$ These studies suggested that the contractile characteristics of the diaphragm were similar to those of other skeletal muscles. However, there has been no in vivo study in man. Techniques are now well established for measuring the force of isometric contraction of a large proximal muscle (quadriceps) and a small distal muscle (adductor pollicis) after electrical stimulation at different frequencies-the frequency-force curve. ${ }^{4}$ The same technique has been used to investigate the contractile properties of an accessory muscle of respiration-the sternomastoid. $^{5}$ It is not possible to measure directly the force produced by the contraction of the diaphragm and the best available index of this force is transdiaphragmatic pressure (Pdi). We therefore studied Pdi in response to stimulation of the phrenic nerve in man.

The essential function of muscle is to generate force and thus fatigue can be defined as failure to sustain a given force. ${ }^{6}$ In patients with lung disease the diaphragm works against a greatly increased load, and it has been proposed that fatigue of

Address for reprint requests: Dr Malcolm Green, Brompton Hospital, Fulham Road, London SW3 6HP. the diaphragm and other respiratory muscles is an important factor in respiratory failure. ${ }^{7}$ The nature of such postulated fatigue in the diaphragm has not yet been characterised. When normal subjects breathe through inspiratory resistances the endurance of the diaphragm is a function of the load as in other skeletal muscles. ${ }^{8} \mathrm{~A}$ technique for predicting muscle fatigue based on analysis of the electromyographic power spectrum ${ }^{910}$ has been modified to investigate possible fatigue in the diaphragm. ${ }^{11}$ It appears that the electromyographic changes occur before the diaphragm fails as a pressure generator.

When skeletal muscles sustain a high load for a prolonged period their contractile properties are altered and can remain so for several hours. In particular forces generated by electrical stimulation at low frequencies are reduced and thus the frequency-force curve of the muscle becomes shifted to the right; this has been called "low frequency fatigue." 12 Normal subjects can develop low frequency fatigue in the sternomastoid muscle after breathing through an inspiratory resistance. ${ }^{5}$ We therefore restudied the Pdi in response to phrenic stimulation after inspiratory loads to see if the diaphragm also developed low frequency fatigue.

\section{Methods}

The subjects were three normal men, aged 25-35 years, with normal respiratory function and no 
muscle weakness. To ensure maximum relaxation they were studied upright, leaning backwards on a board inclined at $10^{\circ}$ to the vertical, with their arms resting on supports.

\section{PHRENIC NERVE STIMULATION}

A reference electrode (anode) was applied to the manubrium. The right phrenic nerve was stimulated in the root of the neck immediately posterior to the sternomastoid muscle using a surface probe electrode (cathode), $5 \mathrm{~mm}$ in diameter, covered with a saline-soaked cloth (fig 1). This caused contraction of the right hemidiaphragm. During electrical stimulation the electrode was held firmly in position by the subject who was being studied. The phrenic nerve was stimulated with unidirectional square wave impulses of $100 \mu$ s duration and 20-50 volts, in a continuous sequence at $1,10,20,50$, and $100 \mathrm{~Hz}$. Stimulation was for two seconds at each frequency and the complete sequence was repeated several times during each experimental session and also on separate days, to test the reproducibility of the frequency-pressure relationship. In order to ensure phrenic nerve stimulation was similar before and after loading, the electromyogram (EMG) was recorded by means of surface disc electrodes $1 \mathrm{~cm}$ diameter placed in the right sixth and seventh intercostal

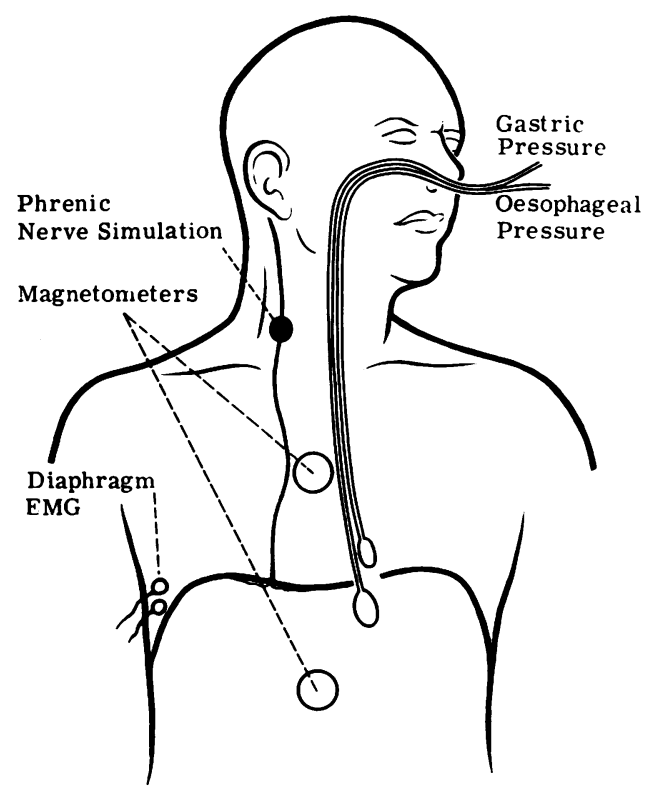

Fig 1 Method of recording transdiaphragmatic pressure during stimulation of the right phrenic nerve (see text). spaces $3 \mathrm{~cm}$ from the costal margin. During stimulation of the phrenic nerve there is contraction of the local neck muscles and the electrode can be displaced from the phrenic nerve, particularly at high stimulation frequencies. If the electrode loses contact with the nerve the diaphragm EMG fades and the EMG is therefore an important indication that excitation of the diaphragm has been satisfactory. Results that showed any fade of diaphragmatic EMG were discarded.

\section{TRANSDIAPHRAGMATIC PRESSURE}

Gastric pressure (Pg) and oesophageal pressure (Poes) were measured using balloons passed through the nose into the stomach and lower oesophagus. ${ }^{13} 14$ Transdiaphragmatic pressure was derived by subtracting Poes from $\mathrm{Pg}$ and was defined as zero at resting end-expiration.

Magnetometers were used to ensure that the shape and length - that is, muscle fibre lengthof the diaphragm was constant during phrenic nerve stimulation. Two pairs of magnetometers were applied to the chest wall, anteriorly and posteriorly, one pair at the level of the lower end of the sternum and the other pair at the level of the umbilicus. The output of the magnetometers was displayed on a large oscilloscope in front of the subject so that the shape of the ribcage and abdomen (and hence the diaphragm) could be returned to exactly the same configuration for each phrenic stimulation. To minimise diaphragmatic shortening, and thereby ensure isometric contraction, the abdomen was strapped and the glottis kept closed during stimulation.

The data from the EMG, magnetometers, gastric, oesophageal, and transdiaphragmatic pressures were displayed on a monitor oscilloscope, recorded on a tape recorder (Racal Store 7) and subsequently played back onto a paper recorder.

INSPIRATORY LOADING

To investigate the effect of increased work on the contractile properties of the diaphragm the frequency-pressure characteristics were established and then the subjects undertook a period of inspiratory loading. An inspiratory resistance was selected for each patient such that he generated $70 \%$ of his maximum voluntary Pdi with each breath; expiration was not loaded. Pdi was displayed on an oscilloscope in front of the subjects with the $70 \%$ Pdi marked. The subject was asked to sustain $70 \%$ Pdi throughout inspiration, but could select whatever tidal volume, respiratory 
rate, and pattern of breathing he found necessary to achieve this result. Each subject was studied on more than one occasion and the time for which the target could be sustained varied from one test to the next and between subjects, ranging from 10-20 minutes. Loaded inspiration was stopped when the target could no longer be reached for three out of four breaths. After the subject had rested for five minutes the contractile properties of the diaphragm were re-examined. The results are expressed in each case as the mean \pm standard deviation calculated by Student's $t$ test.

\section{Results}

CONTRACTILE PROPERTIES: THE FREQUENCY-PDI CURVE

The normal frequency-Pdi curve for the fresh diaphragm was constructed from three stimulation sequences on each of the three subjects (fig 2).

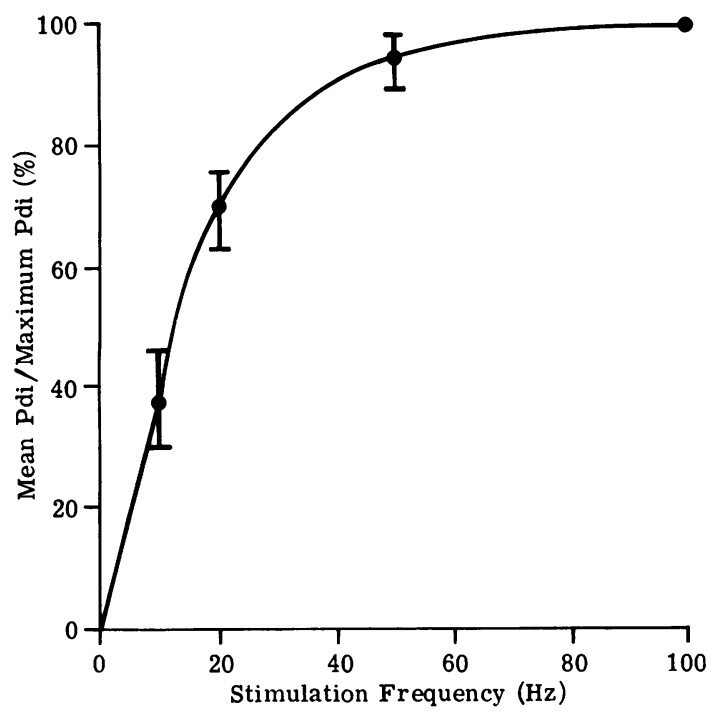

Fig 2 Frequency-Pdi curve of the diaphragm for three normal subjects each tested three times. The Pdi of each subject is expressed as a percentage of his maximal stimulated Pdi.

Transdiaphragmatic pressure rose rapidly at low stimulation frequencies and reached a plateau at $40-60 \mathrm{~Hz}$. A useful index of the contractile response to electrical stimulation is given by the pressure at $20 \mathrm{~Hz}$ as a percentage of that at $50 \mathrm{~Hz}$. This value in the diaphragm was $69.8 \% \pm 3.8 \%$ (mean $\pm \mathrm{SD}, \mathrm{n}=15$ ).

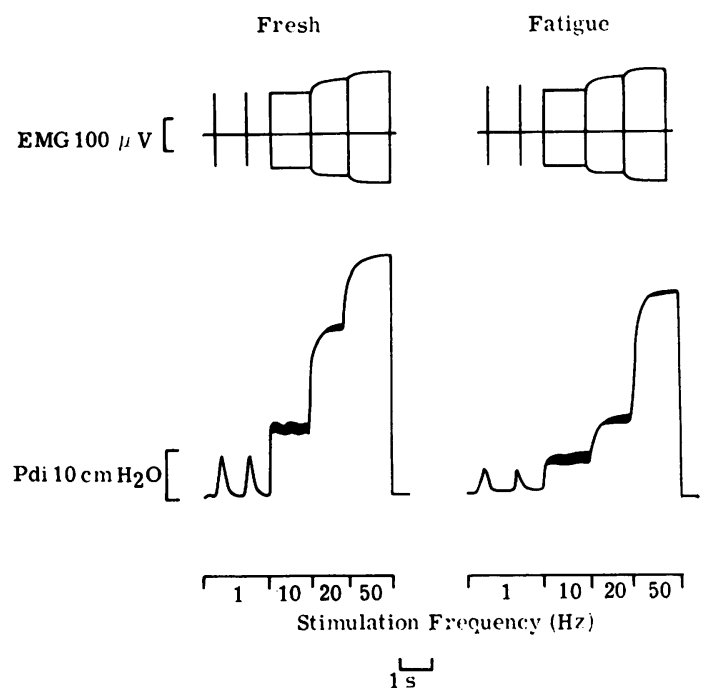

Fig 3 Profile of EMG (above) and Pdi (below) in response to phrenic nerve stimulation from 1 to $50 \mathrm{~Hz}$ in one subject. Left: before fatigue; right: after inspiratory load showing low frequency fatigue.

LOW FREQUENCY FATIGUE

The generation of $70 \%$ maximum inspiratory Pdi for as long as possible produced low frequency fatigue of the diaphragm (fig 3 ) in all three subjects. The loaded runs were performed more than once on each person and numerous results from diaphragms when fresh and after loads were available for comparison. Such a comparison of five frequency-Pdi curves before and after loading for each of the three subjects demonstrated a highly significant $(p<0.001)$ fall in the Pdi at $20 \mathrm{~Hz}$ as a percentage of Pdi at $50 \mathrm{~Hz}$ from $69.8 \% \pm$ $3.8 \%$ (mean $\pm \mathrm{SD}, \mathrm{n}=15$ ) to $48 \cdot 7 \% \pm 7.0 \%$.

\section{Discussion}

\section{CONTRACTILE PROPERTIES}

We have demonstrated that the frequency-pressure curve of the diaphragm is essentially the same as the frequency-force curve of other skeletal muscles (fig 4). The ratio of muscle force at $20 \mathrm{~Hz}$ to that at $50 \mathrm{~Hz}$ is $75.6 \pm 6.3 \%$ for quadriceps, $73 \cdot 1 \pm 7 \cdot 8 \%$ for adductor pollicis, ${ }^{4}$ and $80 \cdot 5 \pm 9 \cdot 2 \%$ for sternomastoid, ${ }^{5}$ and these results are similar to that of the diaphragm $(69 \cdot 8 \% \pm 3 \cdot 8 \%)$. Force generation in any muscle is determined by the number of contractile units recruited and the stimulation frequency. It is unlikely that the voltage applied to the phrenic nerve was supramaximal, because of overlying tissues. However, it is known from 


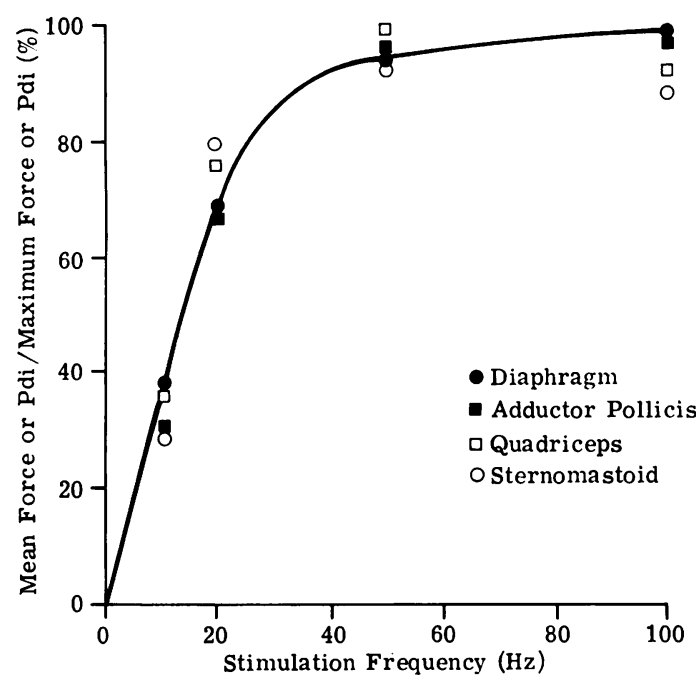

Fig 4 Frequency-Pdi curve of the diaphragm and frequency-force curves of sternomastoid, quadriceps, and adductor pollicis, expressed as a percentage of the stimulated maximum for each muscle.

studies on the quadriceps muscle that when a proportion of muscle fibres are stimulated the characteristics defined are similar to those derived from stimulation of every muscle fibre. ${ }^{4}$ The shape of the frequency-force curve is of fundamental importance for the development of tension and it is interesting that the curve is the same shape in the diaphragm as other muscles. This observation makes it likely that much of our knowledge concerning force generation can be applied with confidence to this respiratory muscle. Although there are no direct data on respiratory muscles, there is good evidence from limb muscles to suggest that during day-to-day activities the motor neurone firing frequency is low, between $5-30 \mathrm{~Hz}$, and therefore on the steep part of the frequencyforce curve. ${ }^{6}$ Although higher firing frequencies can be achieved for brief periods, even with maximum voluntary effort the frequency is not sustained above $30 \mathrm{~Hz}$ for more than a few seconds. ${ }^{15}$ Thus, if the shape of the frequency-pressure curve of the diaphragm were altered so that pressure generation at low frequencies was impaired, there is likely to be a substantial reduction in inspiratory performance at the normal output of the respiratory centre.

\section{FATIGUE}

It seemed possible that the diaphragm might be resistant to fatigue because of its constant activity throughout life. However, this study illustrates that the diaphragm is similar to other muscles in that it can develop low frequency fatigue. This fatigue of the diaphragm is in keeping with earlier studies that have shown low frequency fatigue in the sternomastoid muscles both in normal subjects after inspiratory loading, ${ }^{5}$ and in patients with obstructive lung disease after sustained maximum voluntary ventilation. ${ }^{16}$

The precise mechanism of low frequency fatigue is not known. It is characteristic of this type of muscle fatigue that the excitation of muscle is normal whereas the contractile response is reduced. When low frequency fatigue is present the muscle EMG is entirely normal and therefore this type of fatigue is not detected by techniques that measure shifts of the EMG power spectrum. ${ }^{17}$

The demonstration of low frequency fatigue of the diaphragm in normal subjects suggests that patients with respiratory disease may develop the same type of fatigue. Respiratory muscle work is greater in obstructive lung disease because airways resistance is increased and hyperinflation causes the chest wall to be on a less compliant part of its pressure-volume characteristic. Hyperinflation makes the action of the respiratory muscles less effective and by shortening the muscles adversely affects their length-tension characteristics. In restrictive lung diseases muscle work is increased because of the large fall in lung compliance. In both groups of patients the muscles have to sustain large loads for prolonged periods and with no opportunity for rest. At the same time as increased demands are made there may be arterial hypoxaemia reducing the supply of oxygen to the working muscle.

If low frequency fatigue were to occur in the diaphragm of patients with respiratory disease, ventilatory failure could develop or be intensified. To maintain force production and therefore ventilation in these circumstances the respiratory drive must be increased. However it seems likely that there is a limit to the output of the respiratory motor neurones as is the case for other muscles. Such a limit would result in a fall in force production and a reduction in ventilation. Development of less marked low frequency fatigue might be associated with ventilation being maintained at the cost of maximal or near maximal respiratory drive. In this situation depression of respiratory drive and motor neurone firing frequency-for example, by sedatives or inappropriate oxygen therapy-could cause a substantial fall in muscle force and precipitate ventilatory failure. Since low frequency fatigue can persist 
for many hours, ${ }^{5} 12$ this may explain why a period of assisted ventilation may be useful in allowing the muscles time to rest and return to a normal contractile state.

We would like to thank Dr Alan Henderson for experimental assistance and Miss $\mathrm{H}$ Whippy for kindly typing the manuscript. Support from Muscular Dystrophy Group of Great Britain is also acknowledged gratefully.

\section{References}

1 Adrian ED, Bronk DW. The discharge of impulses in motor nerve fibres. Part I. Impulses in single fibres of the phrenic nerve. J Physiol 1928; 66:81-101.

2 Moulds RFW, Young A, Jones DA, Edwards RHT. A study of the contractility, biochemistry and morphology of an isolated preparation of human skeletal muscle. Clin Sci 1977; 52:291-7.

3 Edwards RHT. The diaphragm as a muscle. Mechanisms underlying fatigue. Am Rev Respir Dis 1979; 119: pt 2 suppl, 81-4.

4 Edwards RHT, Young A, Hoskin GP, Jones DA. Human skeletal muscle function: description of tests and normal values. Clin Sci 1977; 52: 283-90.

5 Moxham J, Wiles CM, Newham D, Edwards RHT. Sternomastoid muscle function and fatigue in man. Clin Sci 1980; 59:463-8.

6 Edwards RHT. Physiological analysis of skeletal muscle weakness and fatigue. Clin Sci 1978; 54: 463-70.

7 Macklem PT, Roussos CS. Respiratory muscle fatigue: a cause of respiratory failure? Clin Sci 1977; 53:419-22.

8 Roussos CS, Macklem PT. Diaphragmatic fatigue in man. J Appl Physiol 1977; 43:189-97.

9 Kogi K, Hakamada T. Slowing of surface electromyogram and muscle strength in muscle fatigue. Reports of the Institute for Science of Labour (Japan) 1962; 60:27-42.

10 Lindstrom L, Magnusson R, Peterson I. Muscular fatigue and action potential conduction velocity changes studied with frequency analysis of EMG signals. Electromyography 1970; 10:341-56.

11 Gross D, Grassino A, Macklem PT. Electromyogram pattern of diaphragmatic fatigue. $J$ Appl Physiol 1979; 46:1-7.

12 Edwards RHT, Hill DK, Jones DA, Merton PA. Fatigue of long duration in human skeletal muscle after exercise. $J$ Physiol 1977; 272:769-78.

13 Milic-Emili J, Mead J, Turner JM, Glauser EM. Improved technique for estimating pleural pressure from oesophageal balloons. J Appl Physiol 1964; 19:207-11.

14 Agostini E, Rahn H. Abdominal and thoracic pressures at different lung volumes. J A ppl Physiol 1960; 15:1087-92.

15 Jones DA, Bigland-Ritchie B, Edwards RHT. Excitation frequency and muscle fatigue: mechanical responses during voluntary and stimulated contractions. Exp Neurol 1979; 64:401-13.

16 Moxham J, Spiro SG, Wiles CM, Edwards RHT. Respiratory muscle function and fatigue: studies on the sternomastoid. Thorax 1980; 35:226.

17 Moxham J, DeTroyer A, Farkas G, Macklem PT. Edwards R, Roussos C. Relationship of EMG power spectrum (PS) with low and high frequency fatigue in human muscle. Physiologist 1979; 22: 91-9. 